\title{
Correction to: Relationship between sagittal balance and adjacent segment disease in surgical treatment of degenerative lumbar spine disease: meta-analysis and implications for choice of fusion technique
}

\author{
Kevin Phan ${ }^{1}$ - Alexander Nazareth ${ }^{2}$. Awais K. Hussain ${ }^{3}$ - Adam A. Dmytriw ${ }^{4} \cdot$ Mithun Nambiar $^{5}$ - Damian Nguyen ${ }^{6}$. \\ Jack Kerferd ${ }^{1}$. Steven Phan ${ }^{1}$. Chet Sutterlin III $^{7}$. Samuel K. Cho ${ }^{3} \cdot$ Ralph J. Mobbs ${ }^{1}$
}

Published online: 13 October 2021

๑) Springer-Verlag GmbH Germany, part of Springer Nature 2021

\section{Correction to: European Spine Journal (2018) 27:1981-1991, https://doi.org/10.1007/s00586-018-5629-6}

The previous version was missing citations to studies analyzed in the meta-analysis and listed in Table 1. They are given below:

Alentado, V. J., Lubelski, D., Healy, A. T., Orr, R. D., Steinmetz, M. P., Benzel, E. C., \& Mroz, T. E. (2016). Predisposing characteristics of adjacent segment disease after lumbar fusion. Spine, 41(14), 1167-1172.

Anandjiwala, J., Seo, J. Y., Ha, K. Y., Oh, I. S., \& Shin, D. C. (2011). Adjacent segment degeneration after instrumented posterolateral lumbar fusion: a prospective cohort study with a minimum five-year follow-up. European Spine Journal, 20(11), 1951-1960.

The original article can be found online at https://doi.org/10.1007/ s00586-018-5629-6.

Kevin Phan

kphan.vc@gmail.com

1 NeuroSpine Surgery Research Group (NSURG), Prince of Wales Private Hospital, Sydney, Australia

2 Keck School of Medicine, University of Southern California, Los Angeles, USA

3 Department of Orthopaedics Surgery, Icahn School of Medicine at Mount Sinai, New York, NY 10029, USA

4 Department of Medical Imaging, University of Toronto, Toronto, Canada

5 Department of Surgery, University of Melbourne, Melbourne, Australia

6 University of Adelaide, Adelaide, Australia

7 Spinal Health International, Inc, Longboat Key, FL, USA
Di Martino, A., Quattrocchi, C. C., Scarciolla, L., Papapietro, N., Zobel, B. B., \& Denaro, V. (2014). Estimating the risk for symptomatic adjacent segment degeneration after lumbar fusion: analysis from a cohort of patients undergoing revision surgery. European Spine Journal, 23(6), 693-698.

Matsumoto, T., Okuda, S., Maeno, T., Yamashita, T., Yamasaki, R., Sugiura, T., \& Iwasaki, M. (2017). Spinopelvic sagittal imbalance as a risk factor for adjacent-segment disease after single-segment posterior lumbar interbody fusion. Journal of Neurosurgery: Spine, 26(4), 435-440.

Nakashima, H., Kawakami, N., Tsuji, T., Ohara, T., Suzuki, Y., Saito, T., ... \& Imagama, S. (2015). Adjacent segment disease after posterior lumbar interbody fusion. Spine, 40(14), E831-E841.

Rothenfluh, D. A., Mueller, D. A., Rothenfluh, E., \& Min, K. (2015). Pelvic incidence-lumbar lordosis mismatch predisposes to adjacent segment disease after lumbar spinal fusion. European Spine Journal, 24(6), 1251-1258. Wang, H., Ma, L., Yang, D., Wang, T., Liu, S., Yang, S., \& Ding, W. (2017). Incidence and risk factors of adjacent segment disease following posterior decompression and instrumented fusion for degenerative lumbar disorders. Medicine, 96(5).

Yamasaki, K., Hoshino, M., Omori, K., Igarashi, H., Nemoto, Y., Tsuruta, T., ... \& Matsuzaki, H. (2017). Risk factors of adjacent segment disease after transforaminal inter-body fusion for degenerative lumbar disease. Spine, 42(2), E86-E92.

Publisher's Note Springer Nature remains neutral with regard to jurisdictional claims in published maps and institutional affiliations. 